\title{
LIFTING FIXED POINTS OF COMPLETELY POSITIVE SEMIGROUPS
}

\author{
BEBE PRUNARU
}

\begin{abstract}
Let $\left\{\phi_{s}\right\}_{s \in S}$ be a commutative semigroup of completely positive, contractive, and weak*-continuous linear maps acting on a von Neumann algebra $N$. Assume there exists a semigroup $\left\{\alpha_{s}\right\}_{s \in S}$ of weak*-continuous *endomorphisms of some larger von Neumann algebra $M \supset N$ and a projection $p \in M$ with $N=p M p$ such that $\alpha_{s}(1-p) \leq 1-p$ for every $s \in S$ and $\phi_{s}(y)=p \alpha_{s}(y) p$ for all $y \in N$. If $\inf _{s \in S} \alpha_{s}(1-p)=0$ then we show that the map $E: M \rightarrow N$ defined by $E(x)=p x p$ for $x \in M$ induces a complete isometry between the fixed point spaces of $\left\{\alpha_{s}\right\}_{s \in S}$ and $\left\{\phi_{s}\right\}_{s \in S}$.
\end{abstract}

Let $(S,+, 0)$ be a commutative semigroup with unit 0 . Consider the partial preorder on $S$ induced by the semigroup structure as follows. If $s, t \in S$ then $s \leq t$ if and only if there exists $r \in S$ such that $s+r=t$. If $X$ is a Hausdorff topological space and $f: S \rightarrow X$ is a function, , then $\lim _{s \in S} f(s)$ denotes its limit along the directed set $(S, \leq)$, whenever this limit exists.

Let $M$ be a von Neumann algebra. Let $C P(M)$ denote the semigroup of all completely positive, contractive and weak*-continuous linear maps $\beta: M \rightarrow M$. Let also $\operatorname{End}(M)$ be the semigroup of all weak*-continuous ${ }^{*}$-endomorphisms of $M$. A family $\left\{\beta_{s}\right\}_{s \in S} \subset C P(M)$ is called a semigroup if the map $s \mapsto \beta_{s}$ is a unital homomorphism of semigroups from $S$ into $C P(M)$.

Suppose now that $\left\{\alpha_{s}\right\}_{s \in S} \subset \operatorname{End}(M)$ is a semigroup. Let $p$ be an orthogonal projection in $M$ such that

$$
\alpha_{s}(1-p) \leq 1-p \quad \forall s \in S
$$

Then one can define, for every $s \in S$, a completely positive mapping on the von Neumann algebra $N=p M p$ as follows:

$$
\phi_{s}(x)=p \alpha_{s}(x) p \quad \forall x \in N .
$$

It is clear that $\left\{\phi_{s}\right\}_{s \in S} \subset C P(N)$. A short calculation shows that

$$
\phi_{s}(p x p)=p \alpha_{s}(x) p \quad \forall x \in M,
$$

and using this, one can show that $\left\{\phi_{s}\right\}_{s \in S}$ is a semigroup. According to the terminology used in Chapter 8 of [1, where this construction is given for one-parameter semigroups, $\left\{\alpha_{s}\right\}_{s \in S}$ is a dilation of $\left\{\phi_{s}\right\}_{s \in S}$ and $p$ is a co-invariant projection for $\left\{\alpha_{s}\right\}_{s \in S}$.

We shall prove the following result, which shows that, under a suitable minimality condition, the fixed point spaces of $\left\{\alpha_{s}\right\}_{s \in S}$ and $\left\{\phi_{s}\right\}_{s \in S}$ are completely isometric. We point out that the minimality condition is always satisfied by the minimal Edilation of a CP-semigroup as constructed in [1.

2000 Mathematics Subject Classification. Primary 46L55, Secondary 46L05.

Key words and phrases. dilation theory, completely positive semigroups, fixed points. 
Theorem 1. Let $M \subset B(H)$ be a von Neumann algebra on some Hilbert space $H$. Let $(S,+, 0)$ be a commutative semigroup with unit and let $\left\{\alpha_{s}\right\}_{s \in S} \subset \operatorname{End}(M)$ be a semigroup of weak*-continuous ${ }^{*}$-endomorphisms of $M$. Let $p \in M$ be a projection such that

$$
\alpha_{s}(1-p) \leq 1-p \quad \forall s \in S
$$

and

$$
\inf _{t \in S} \alpha_{t}(1-p)=0 .
$$

Let $\left\{\phi_{s}\right\}_{s \in S} \subset C P(N)$ be the compression of $\left\{\alpha_{s}\right\}_{s \in S}$ to $N=p M p$ defined by

$$
\phi_{s}(x)=p \alpha_{s}(x) p \quad \forall x \in N .
$$

Let

$$
M^{\alpha}=\left\{x \in M: \alpha_{t}(x)=x, \forall t \in S\right\}
$$

and

$$
N^{\phi}=\left\{x \in N: \phi_{t}(x)=x, \forall t \in S\right\}
$$

and let $C^{*}\left(N^{\phi}\right)$ be the $C^{*}$-subalgebra of $N$ generated by $N^{\phi}$. Let $E: M \rightarrow M$ be defined by

$$
E(x)=p x p \quad \forall x \in M .
$$

Then the following hold true:

(1) For each $y \in C^{*}\left(N^{\phi}\right)$ there exists the limit (in the strong operator topology)

$$
\pi(y)=s o-\lim _{s \in S} \alpha_{s}(y)
$$

and the map $y \mapsto \pi(y)$ is a ${ }^{*}$-homomorphism from $C^{*}\left(N^{\phi}\right)$ onto $M^{\alpha}$ such that $(\pi \circ E)(x)=x$ for all $x \in M^{\alpha}$.

(2) E induces a complete isometry between $M^{\alpha}$ and $N^{\phi}$.

(3) For each $y \in C^{*}\left(N^{\phi}\right)$ there exists the limit

$$
\Phi(y)=s o-\lim _{s \in S} \phi_{s}(y)
$$

and the map $y \mapsto \Phi(y)$ is completely positive, idempotent, $\operatorname{Ran}(\Phi)=N^{\phi}$, and $E \circ \pi=\Phi$ on $C^{*}\left(N^{\phi}\right)$.

Proof. First,we show that $E\left(M^{\alpha}\right)=N^{\phi}$. It is clear that $E\left(M^{\alpha}\right) \subset N^{\phi}$. Let $\mu$ be an invariant mean on $S$. This means that $\mu$ is a state on the von Neumann algebra $\ell^{\infty}(S)$ of all complex-valued bounded functions on $S$ that remains invariant under translations. It is well known [5] that any commutative semigroup is amenable, in the sense that it admits invariant means.

Let $y \in N^{\phi}$. For each $\gamma$ in the predual $M_{*}$ of $M$, let $f_{\gamma} \in \ell^{\infty}(S)$ be defined by

$$
f_{\gamma}(s)=\left(\alpha_{s}(y), \gamma\right) \quad s \in S .
$$

Then there exists $z \in M$ such that

$$
(z, \gamma)=\mu\left(f_{\gamma}\right) \quad \forall \gamma \in M_{*} .
$$

Since $\alpha_{s}$ are weak ${ }^{*}$ continuous and $\left\{\alpha_{s}\right\}_{s \in S}$ is a semigroup, it follows that $z \in M^{\alpha}$. Moreover $p z p=y$ and this shows that $E\left(M^{\alpha}\right)=N^{\phi}$.

In order to go further, we need to use the minimality assumption on $\left\{\alpha_{s}\right\}_{s \in S}$. Suppose now that $w \in M^{\alpha}$. Since $\inf _{t \in S} \alpha_{t}(1-p)=0$ we see that

$$
s o-\lim _{s \in S} \alpha_{s}(p w p)=w \text {. }
$$


Since $E\left(M^{\alpha}\right)=N^{\phi}$ it follows that the limit

$$
\pi(y)=s o-\lim _{s \in S} \alpha_{s}(y)
$$

exists for every $y \in N^{\phi}$ and that $\pi \circ E=i d$ on $M^{\alpha}$. In particular $E$ is completely isometric on $M^{\alpha}$. All the other assertions are straightforward consequences of what we have already proved.

This result and its proof provide, in particular, an alternate and simplified approach to the lifting theorem for fixed points of completely positive maps from $[\underline{6}$. In the case when $S$ is either a commutative, countable and cancellative semigroup or $S=\mathbb{R}_{+}^{d}$ for some $d \geq 1$, and $\left\{\alpha_{s}\right\}_{s \in S}$ are unit preserving, part 2 of Theorem 1 follows directly from Proposition 4.4 together with Theorem 4.5 from [4. In the case when $\left\{\phi_{s}\right\}_{s \in S}$ is the semigroup induced by the unilateral shift on the Hardy space $H^{2}$, the existence of the limit in part 3 of Theorem 1 is proved in [2].

We close with the following result which shows that part 3 of the previous theorem holds true even without assuming the existence of a dilation.

Theorem 2. Let $N \subset B(H)$ be a von Neumann algebra on some Hilbert space $H$. Let $(S,+, 0)$ be a commutative semigroup with unit. Let $\left\{\phi_{s}\right\}_{s \in S}$ be a semigroup of completely positive, contractive and weak*-continuous linear maps on $N$. Let

$$
N^{\phi}=\left\{x \in N: \phi_{t}(x)=x, \forall t \in S\right\}
$$

and let $C^{*}\left(N^{\phi}\right)$ be the $C^{*}$-subalgebra of $N$ generated by $N^{\phi}$. Then for each $y \in$ $C^{*}\left(N^{\phi}\right)$ there exists the strong-operator limit

$$
\Phi(y)=s o-\lim _{s \in S} \phi_{s}(y)
$$

and the map $y \mapsto \Phi(y)$ is completely positive, contractive, idempotent, and moreover $\operatorname{Ran}(\Phi)=N^{\phi}$.

Proof. Let $\mu$ be an invariant mean on $S$. Let $y \in N$. For each $\gamma$ in the predual $N_{*}$ of $N$, let $f_{\gamma} \in \ell^{\infty}(S)$ be defined by

$$
f_{\gamma}(s)=\left(\phi_{s}(y), \gamma\right) \quad s \in S .
$$

Then there exists $z \in N$ such that

$$
(z, \gamma)=\mu\left(f_{\gamma}\right) \quad \forall \gamma \in N_{*} .
$$

Since $\left\{\phi_{s}\right\}_{s \in S}$ are weak* continuous and $\left\{\phi_{s}\right\}_{s \in S}$ is a semigroup, it follows that $z \in N^{\phi}$. Let us denote $z=\rho(y)$. The mapping $\rho: N \rightarrow N$ is completely positive, contractive, idempotent, and $\operatorname{Ran}(\rho)=N^{\phi}$. Moreover

$$
\phi_{s} \circ \rho=\rho \circ \phi_{s}=\rho .
$$

Let $\Phi: C^{*}\left(N^{\phi}\right) \rightarrow N^{\phi}$ be the restriction of $\rho$ to $C^{*}\left(N^{\phi}\right)$. A well known result from 3 shows that

$$
\Phi(\Phi(x) y)=\Phi(\Phi(x) \Phi(y)) \quad \forall x, y \in C^{*}\left(N^{\phi}\right) .
$$

This easily implies that $\operatorname{ker}(\Phi)$ is the closed left ideal in $C^{*}\left(N^{\phi}\right)$ generated by all the operators of the form $x y-\Phi(x y)$ with $x, y \in N^{\phi}$. Moreover by polarization we see that $\operatorname{ker} \Phi$ is the closed ideal of $C^{*}\left(N^{\phi}\right)$ generated by all the operators of the form $x^{*} x-\Phi\left(x^{*} x\right)$ with $x \in N^{\phi}$. 
Let $x \in N^{\phi}$. Since $\left\{\phi_{s}\right\}_{s \in S}$ are completely positive, they satisfy the KadisonSchwarz inequality, therefore

$$
\phi_{s}\left(x^{*} x\right)-x^{*} x \geq 0
$$

for all $s \in S$ hence the net $\left\{\phi_{s}\left(x^{*} x\right)\right\}_{s \in S}$ is monotone increasing. It follows from the way $\Phi$ is constructed that

$$
\Phi\left(x^{*} x\right)=s o-\lim _{s \in S} \phi_{s}\left(x^{*} x\right) .
$$

Let $y=\Phi\left(x^{*} x\right)-x^{*} x$. It follows that

$$
s o-\lim _{s \in S} \phi_{s}(y)=0 .
$$

Let $a \in C^{*}\left(N^{\phi}\right)$ and let $h \in H$. Then

$$
\left\|\phi_{s}\left(a y^{1 / 2}\right) h\right\|^{2} \leq\left(\phi_{s}\left(y^{1 / 2} a^{*} a y^{1 / 2}\right) h, h\right) \leq\|a\|^{2}\left(\phi_{s}(y) h, h\right) .
$$

This shows that $\lim _{s \in S}\left\|\phi_{s}(z) h\right\|=0$ for every $z \in \operatorname{ker} \Phi$ therefore

$$
\Phi(w)=s o-\lim _{s \in S} \phi_{s}(w)
$$

for every $w \in C^{*}\left(N^{\phi}\right)$. This completes the proof.

\section{REFERENCES}

[1] W. Arveson, Noncommutative dynamics and E-semigroups, Springer Monographs in Mathematics, Springer-Verlag, New York, 2003.

[2] J. Barria and P. R. Halmos, Asymptotic Toeplitz operators. Trans. Amer. Math. Soc. 273 (1982), 621-630.

[3] M.D. Choi, E.G. Effros, Injectivity and operator spaces, J. Functional Analysis, 24 (1977), no. 2, 156-209.

[4] D. Courtney, Asymptotic lifts of UCP semigroups, Ph.D. Dissertation, University of California, Berkeley, 2007.

[5] J. Dixmier, Les moyennes invariantes dans les semi-groupes et leurs applications, Acta Sci. Math. (Szeged) 12 (1950), 585-590.

[6] B. Prunaru, Lifting fixed points of completely positive mappings, J. Math. Anal. Appl. 350 (2009), 333-339.

Institute of Mathematics "Simion Stollow" of the Romanian Academy, P.O. Box 1-764 RO-014700 Bucharest Romania

E-mail address: bebe.prunaru@gmail.com 